\title{
Prolonged use of the Greenland method of treatment of gonorrhoea
}

\author{
A J EVANS,* G D MORRISON, * AND D J PRICE† \\ From the *Department of Venereology, Plymouth General Hospital, and the + Department of Biological \\ Sciences, Plymouth Polytechnic, Plymouth, Devon
}

SUMMARY Of 1081 patients with gonorrhoea treated with probenecid $1 \mathrm{~g}$ orally and benzyl penicillin 5 megaunits intramuscularly, $96 \cdot 7 \%$ were cured and no complications were encountered. This treatment regimen was associated with a pronounced reduction in the proportion of gonococcal strains showing diminished sensitivity to penicillin, from $51 \%$ to between $4 \%$ and $14 \%$ over a period of four years. When this treatment method was abandoned, however, the percentage of less sensitive strains rose to $33 \%$ over the following 18 months.

\section{Introduction}

By 1971 we were becoming seriously worried because $43 \%$ of gonococci isolated in Plymouth showed diminished sensitivity to penicillin in vitro (a zone of inhibition corresponding to a minimum inhibitory concentration (MIC) $<0 \cdot 1 \mu \mathrm{g} / \mathrm{ml}$ ). In 1969 and 1970 our routine treatment for uncomplicated gonorrhoea in men and women had been with co-trimoxazole tablets $B P(4$ tablets initially and then 2 tablets twice daily for five days).

Olsen and Lomholt ${ }^{1}$ reported in 1969 from Greenland that when probenecid $1 \mathrm{~g}$ and benzyl penicillin 5 megaunits had been introduced as the routine treatment for gonorrhoea the proportion of strains less sensitive to penicillin had fallen noticeably and had remained low. Gray et $a l^{2}$ suggested that the use of this regimen nationally might result in a substantial reduction in the prevalence of less sensitive strains.

In the hope of achieving a similar reduction in these less sensitive strains we adopted Olsen's and Lomholt's ${ }^{1}$ method of treatment, which we have called the Greenland method. From February 1971 to May 1977 this was the standard treatment for gonorrhoea in the clinic at Plymouth. For the next six months alternate cases were treated with either cefuroxime ( $1 \mathrm{~g} \mathrm{i.m}$. and $1 \mathrm{~g}$ probenecid orally) or the Greenland method. Throughout 1978 all cases were treated with cefuroxime (either $1.5 \mathrm{~g}$ i.m. or $0.75 \mathrm{~g}$ plus $1 \mathrm{~g}$ probenecid).

Address for reprints: Dr A J Evans, Treatment Clinic, Plymouth General Hospital, Freedom Fields, Plymouth, Devon

Received for publication 10 September 1979
The results with this regimen, particularly the changes in the sensitivity pattern of the gonococci isolated, are reported here.

\section{Patients and methods}

\section{STUDY POPULATION}

Between February 1971 and May 1977, 1925 cases of gonorrhoea (1227 men and 698 women) were treated with the Greenland method. Only patients who were hypersensitive to penicillin, those with disseminated gonococcal infection or salpingitis, and the rare patient who refused injection were excluded from the series.

The age and racial distribution of the patients remained the same throughout each year of the series. The only discernible change over the period was that the male-to-female ratio changed from $1 \cdot 7: 1$ to $1 \cdot 4: 1$.

Of the initial 1925 patients, 844 (632 men and 212 women) were withdrawn from the series or else defaulted or were transferred elsewhere without follow up. The chief reason for withdrawal was because the patient had been given tetracyline for post-gonococcal urethritis during the follow-up period. As a result the withdrawal/default rate is much higher in men $(51 \%)$ than in women $(30 \%)$. There remained therefore 1081 cases $(595$ men and 486 women) for assessment.

\section{DIAGNOSIS}

Gonorrhoea was diagnosed on the finding of intracellular Gram-negative reniform diplococci in stained smears, or on the isolation of Neisseria gonorrhoeae on culture, or on both. Of the 1925 initial cases, gonococci were found in both Gram- 
stained smears and cultures in $1539(80 \%)$-that is, in $84 \%$ of men and $74 \%$ of women. In $297(15 \%)$ cases the diagnosis was made on the basis of positive results to Gram-stained smears only (190 men and 107 women) and in $89(5 \%)$ cases by culture only-13 $(1 \%)$ men and $76(11 \%)$ women.

Specimens for Gram-stain and culture were taken from the urethra in men and from the urethra and cervix in women and from the rectum in all male homosexuals. Throat swabs for culture were taken from all patients who admitted to orogenital contact, from all male homosexuals, and from any patient who was a known contact of gonorrhoea but from whom we had failed to isolate gonococci from other sites.

\section{CULTURE AND SENSITIVITY TESTING}

Culture and sensitivity testing methods and the criteria for distinguishing sensitive from less sensitive strains were as previously reported. ${ }^{3}$ To ensure that these criteria had not changed during the period of this report, the MIC of benzyl penicillin (determined by plate diffusion) for 12 strains was compared with the zone of inhibition around a $1 \cdot 5$ unit disc (Oxoid). No change was shown to have occurred.

\section{TREATMENT}

All patients with gonorrhoea were treated with probenecid $1 \mathrm{~g}$ by mouth followed after about 15 minutes by a single intramuscular injection of benzyl penicillin 5 megaunits dissolved in $6.5 \mathrm{ml}$ of $0.5 \%$ xylocaine to make a final volume of $8 \mathrm{ml}$ (the Greenland method).

Patients were instructed to refrain from sexual intercourse for two weeks after treatment.

\section{FOLLOW UP}

Patients were seen at seven and 14 days after treatment. If any urethral discharge was found at either follow-up attendance in men who had had gonococcal urethritis, urethral smears and cultures were made. If no discharge was present, a two-glass urine specimen was examined and a smear made of any threads in the urine. At each attendance, specimens for Gram stain and culture were taken from the urethra and cervix in women, from the rectum in patients with gonococcal proctitis, and from the throat in patients with gonococcal pharyngitis.

If gonococci were found at any time within two weeks of treatment, and the patient denied further sexual intercourse, treatment was then considered to have failed. If gonococci were found after two weeks then reinfection was assumed to have occurred. No method of assessing which cases relapsed and which were reinfected is infallible; the method used here was suggested 20 years ago by Dallas. ${ }^{4}$

\section{Results}

Of the 1081 cases ( 595 men and 486 women) treated with the Greenland method and followed up at seven and 14 days, gonococci were isolated on culture during the follow-up period from 34 (20 men and 14 women) and two more had positive results only on Gram stain, making 36 failures in all. The cure rate was $96.7 \%$ (men $96.5 \%$, women $97 \%$ ). Of the men, 27 had gonococcal proctitis and two of them were not cured. Five patients had gonococcal pharyngitis and treatment failure occurred in one.

The failure rate was significantly higher $\left(\chi_{1}^{2}=5 \cdot 878, P<0 \cdot 02\right)$ in patients infected with strains of gonococci with reduced sensitivity to penicillin. Of 919 cases followed up, and from whom strains of gonococci which were fully sensitive to penicillin were isolated, 25 were considered to be treatment failures, giving a failure rate of $2 \cdot 7 \%$, whereas of 162 cases followed up who had strains showing reduced sensitivity to penicillin 11 were treatment failures, giving a failure rate of $6 \cdot 8 \%$.

TABLE Penicillin sensitivity of gonococci isolated between 1971 and 1977

\begin{tabular}{|c|c|c|c|c|c|c|}
\hline \multirow[b]{2}{*}{ Sensitivity to penicillin } & \multicolumn{2}{|c|}{ Men } & \multicolumn{2}{|c|}{ Women } & \multicolumn{2}{|c|}{ Total } \\
\hline & No & $\%$ & No & $\%$ & No & $\%$ \\
\hline $\begin{array}{l}\text { Fully sensitive } \\
\text { Diminished } \\
\text { Total }\end{array}$ & $\begin{array}{l}831 \\
162 \\
993\end{array}$ & $\begin{array}{l}84 \\
16\end{array}$ & $\begin{array}{r}490 \\
74 \\
564\end{array}$ & $\begin{array}{l}87 \\
13\end{array}$ & $\begin{array}{r}1321 \\
236 \\
1557\end{array}$ & $\begin{array}{l}85 \\
15\end{array}$ \\
\hline
\end{tabular}

\section{ANTIBIOTIC SENSITIVITIES}

In 1628 cases gonococci were cultured from at least one site. In 71 cases the isolate died on subculture and antibiotic sensitivity could not therefore be assessed. Results of sensitivity testing to penicillin in the remaining 1557 cases (993 men and 564 women) are given in the Table. This shows that during the six years when the Greenland method was the routine treatment for gonorrhoea $15 \%$ of the isolates showed diminished sensitivity to penicillin. No $\beta$-lactamaseproducing strains were found.

Since 1969 we have followed the variations in the percentage of strains of gonococci which showed diminished sensitivity to penicillin. These variations are shown for each six-month period in the Figure, together with the $95 \%$ confidence limits for a proportion calculated with a correction for continuity thus:

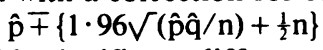

There is a highly significant difference $\left(\chi_{2}^{2}=67 \cdot 734\right.$, $\mathrm{P}<0.001)$ in the proportion of resistant strains between the three different treatment methods.

From mid-1969 to the beginning of 1971, while cotrimoxazole was the routine treatment, the percentage of less sensitive strains rose from 34 to $43 \%$. 
However the difference between the six-month periods is not significant $\left(\chi_{2}^{2}=1 \cdot 386, P=0 \cdot 5\right)$. For the first six-month period after the introduction of the Greenland method there was a further rise in the proportion of less sensitive strains to $51 \%$ (Figure); thereafter there was a rapid fall over the next two years to $5 \%$ in 1973. Use of the Greenland method was continued until mid-1977 and during that time the proportion remained low, within the limits of $3.5 \%$ to $12 \cdot 4 \%$. Thus there is a pronounced heterogeneity between these 13 samples, which is highly significant $\left(\chi_{12}^{2}=197 \cdot 251, \mathrm{P}<0 \cdot 001\right)$.

During the six-month period when alternate patients were treated by the Greenland method or with cefuroxime the proportion of less sensitive strains remained low (6\%) (figure). During 1978, when cefuroxime alone was the routine treatment, the proportion of less sensitive strains rose to $25 \%$ and then to $33 \%$. The difference between samples during this 18 -month period is also significant $\left(\chi_{2}^{2}=36 \cdot 447, \mathrm{P}<0 \cdot 001\right)$.

There has been one other change in the sensitivity pattern. Of all the strains of gonococci isolated in $197110 \%$ were resistant to streptomycin, and all the strains in that year which showed reduced sensitivity to penicillin were also resistant to streptomycin. Over the years the combination of reduced sensitivity to penicillin and resistance to streptomycin has become rare. Only two $(0.8 \%)$ strains in 1978 showed resistance to streptomycin.

\section{Discussion}

The satisfactory results obtained with the Greenland method of treatment (a cure rate of $96.7 \%$ ) is in keeping with the findings of the originators' of this

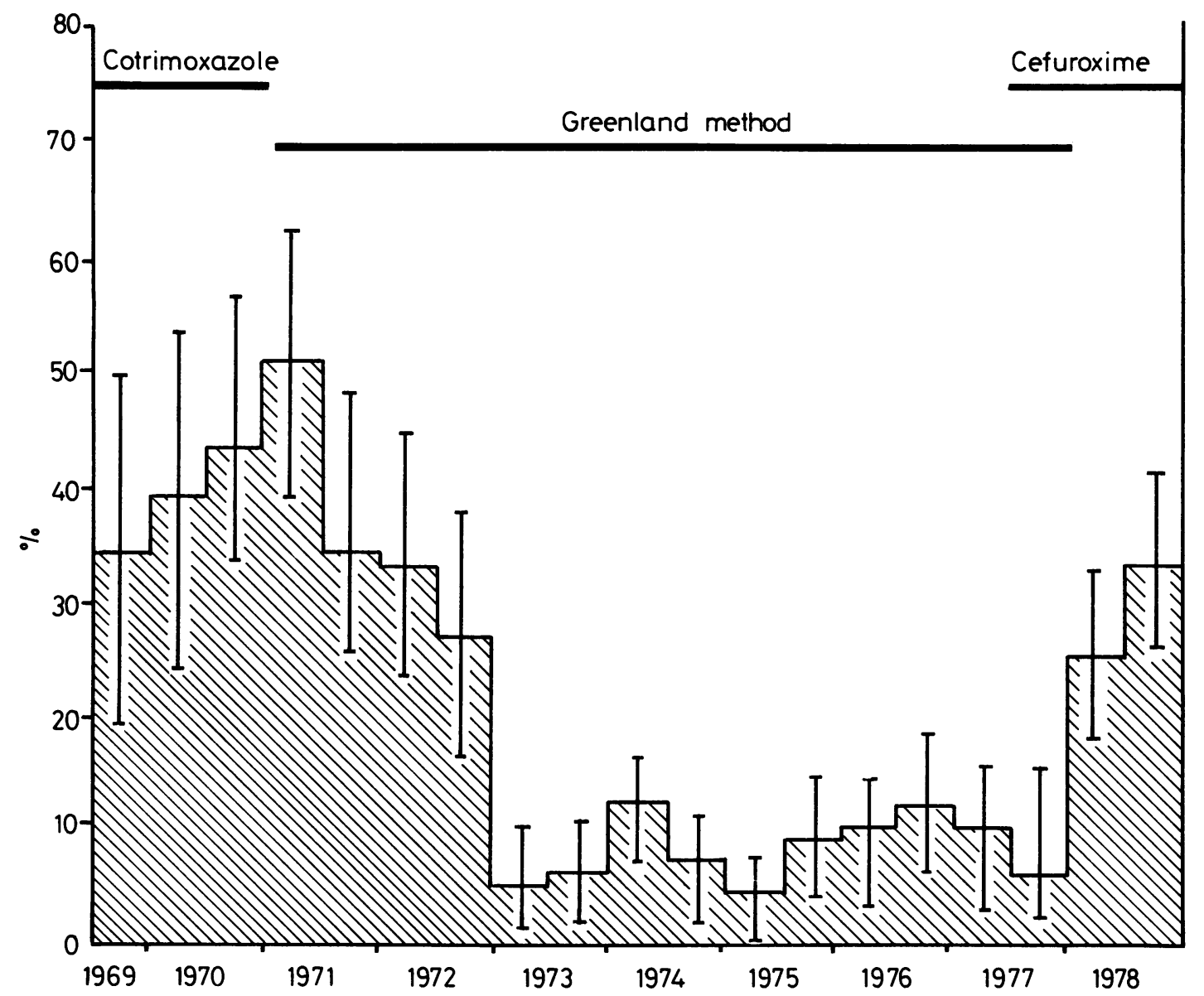

FIG Variation in percentage of strains of gonococci less sensitive to penicillin with different treatment regimens (1969-78) and showing $95 \%$ confidence limits with correction for continuity 
routine and of others. ${ }^{2}$ Wigfield et al ${ }^{5}$ suggested that the large volume $(8 \mathrm{ml})$ of the injection might be disadvantageous. This has not been our experience and we have not recorded any difficulties or major complications with its use.

Olsen and Lomholt ${ }^{1}$ reported a pronounced reduction in Greenland in the proportion of strains of gonococci which were less sensitive to penicillin when their method of treatment was introduced. This improvement continued throughout the $3 \frac{1}{2}$ years covered by their report. Our results from Plymouth confirm these findings, although our series continued for longer (over six years).

What has not previously been reported is that on discontinuing treatment by the Greenland method the proportion of less sensitive strains rises sharply again.

It is difficult to see any reason for these changes. Wigfield $e t a^{5}$ pointed out that the reduction in less sensitive strains could not be attributed to the efficiency of the method, since this would eliminate sensitive and less sensitive strains equally well, and so the proportion of less sensitive strains would remain the same.

There have been many reports of a wide variation in the prevalence of less sensitive strains in different centres, and at the same centres at different times. ${ }^{6-8}$ The statistical analysis however shows that our results are most unlikely to be due to chance and that, whatever the reason, the use of the Greenland method of treatment is associated with a diminution of strains which are less sensitive to penicillin. We know of no other reports associating the therapeutic regimen with variation in the sensitivity pattern of the gonococci isolated.

The near disappearance of streptomycin-resistant strains is surprising. However, streptomycin is now never used for the treatment of gonorrhoea and this may explain the decrease in the number of streptomycin-resistant strains. A similar reduction in streptomycin-resistant strains has been reported from Denmark. ${ }^{9}$

Patients infected with strains of gonococci of reduced sensitivity to penicillin have a higher failure rate when treated with penicillin than do patients infected with fully sensitive strains. ${ }^{6-8} \quad 10-12$ Similar results have been obtained in the present series. Prolonged use of the Greenland method is associated with a reduction in less sensitive strains and should therefore result in a lower failure rate. This theoretical advantage is so great that we have now reverted to the use of the Greenland method as our standard treatment for gonorrhoea in Plymouth. It will be interesting to see if this is again associated with a reduction in the numbers of less sensitive strains of gonococci.

We are indebted to Dr Gillian M Churcher and her staff for carrying out the cultures and antibiotic sensitivities in her department. Our thanks are also due to $\mathrm{Dr} \mathrm{W} \mathrm{H}$ Haskins and to the nursing and secretarial staff of the clinic for their help in this study.

\section{Addendum}

Use of the Greenland method throughout 1979 has been associated with a fall in the proportion of strains less sensitive to penicillin from $33 \%$ to $22 \%$.

\section{References}

1. Olsen GA, Lomholt G. Gonorrhoea treated by a combination of probenecid and sodium penicillin G. Br J Vener Dis 1969: 45: $144-7$

2. Gray RCF, Phillips I, Nicol CS. Treatment of gonorrhoea with three different antibiotic regimens. Br J Vener Dis 1970: 46: 401-3.

3. Evans AJ, Churcher GM, Human RP. Sulphamethoxazoletrimethoprim in the treatment of gonorrhoea. Br J Vener Dis 1972; 48: 179-81.

4. Dallas NL. Penicillin treatment failures in male gonorrhoea. Br J Vener Dis 1958: 34: 194-5.

5. Wigfield AS, Selkon JB, Rich GE. Single-session treatment of uncomplicated gonorrhoea using penicillin combined with cotrimoxazole. Br J Vener Dis 1973: 49: 277-90.

6. Curtis FR, Wilkinson AE. Comparison of the in-vitro sensitivity of gonococci to penicillin with the results of treatment. Br J Vener Dis 1958: 34: 70-82.

7. Medical Research Council Working Party. Resistance of gonococci to penicillin. Lancet 1961: 2: 226-30.

8. Jaffe HW, Biddle JW, Thornberry C, et al. National gonorrhoea therapy monitoring study. In-vitro antibiotic susceptibility and its correlation with treatment results. N Engl J Med 1976: 294: 5-9.

9. World Health Organisation. Neisseria gonorrhoeae and gonococcal infections. WHO Tech Rep Ser 1978: 616: 81.

10. Craddock-Watson JE, Shotter PA, Nicol CS. Sensitivity of strains of gonococci to penicillin, sulphathiazole and streptomycin. Br Med J 1958: 1: 1091-2.

11. Gjessing $\mathrm{HC}$, Odegaard $\mathrm{K}$. Intramuscular injection of procaine penicillin combined with oral administration of chloramphenicol in the treatment of gonorrhoea. Br J Vener Dis 1966: 42: $107-9$.

12. Evans AJ. Relapse of gonorrhoea after treatment with penicillin or streptomycin. Br J Vener Dis 1966: 42: 251-62. 
TO THE EDITOR, British Journal of Venereal Diseases

\section{$\beta$-lactamase-producing gonococci in Hong Kong}

Sir,

From 19 October 1979 to 26 December 1979 15 cases of gonorrhoea were confirmed by culture. Of these 15 cases, three were due to $\beta$-lactamase-producing gonococci. All 15 cases were Chinese patients encountered in private practice.

Specimens for culture were taken with a sterile cottonwool bud from the urethra in men and from the cervix in women and inoculated on to Thayer-Martin media.
These specimens were then transferred to the Department of Extramural Studies of the University of Hong Kong for incubation. The identity of suspected colonies of Neisseria gonorrhoeae was confirmed by the oxidase test, Gramstained smear, and sugar fermentation. $\beta$-lactamase-producing gonococci were detected by the chromogenic cephalosporin test.

A male patient, aged 32 years, presented with dysuria and a purulent urethral discharge. The source of infection was a prostitute in Hong Kong. He was successfully treated with spectinomycin $2 \mathrm{~g}$ intramuscularly.

A female patient, aged 22 years, presented with dysuria and a purulent vaginal discharge. She was a contact of the previous patient and was successfully treated with spectinomycin $2 \mathrm{~g}$ intramuscularly.

A male patient, aged 23 years, presented with dysuria and a purulent urethral discharge. The source of infection was a prostitute in Hong Kong. He was treated with kanamycin $2 \mathrm{~g}$ intramuscularly. Unfortunately the patient did not attend for follow up.

Yours faithfully, F $H$ Wang

Office A, 1/F Fuji Building,

381-383 Lockhart Road,

Hong Kong

\section{Notices}

\section{Project Icarus}

Project Icarus is an independent registered charity producing visual aids for health education. Most of these visual aids are posters and they include a set of eight posters on venereal disease (size 16 in $\times 22$ in). Other sets of posters cover drug abuse, alcoholism, and family planning. Each poster is black and white and is available in single editions or in sets $(£ 2 \cdot 30$ per set or $40 \mathrm{p}$ each including carriage). Further information can be obtained from: Project Icarus Ltd, Raglan House, 4 Clarence Parade, Southsea, Hants PO5 3NU; Tel: Portsmouth (0705) 27460.

\section{Correction}

In the paper by Evans et al on the "Prolonged use of the Greenland method of treatment of gonorrhoea" (1980;56: 88-91) the minimum inhibitory concentration quoted in the first sentence of the introduction should have read $>0.1 \mu \mathrm{g} / \mathrm{ml}$ and not $<0.1 \mu \mathrm{g} / \mathrm{ml}$.

\begin{abstract}
Atlas of sexually transmitted diseases
An "Atlas of Sexually Transmitted Diseases" by Professor E Stolz and Dr J van der Stek, published by Boehringer Ingelheim, is available from the UK company-namely, Boehringer Ingelheim, Southern Industrial Estate, Bracknell, Berks RG12 4YF. Doctors interested in obtaining copies should write direct to the UK company at this address.
\end{abstract}

